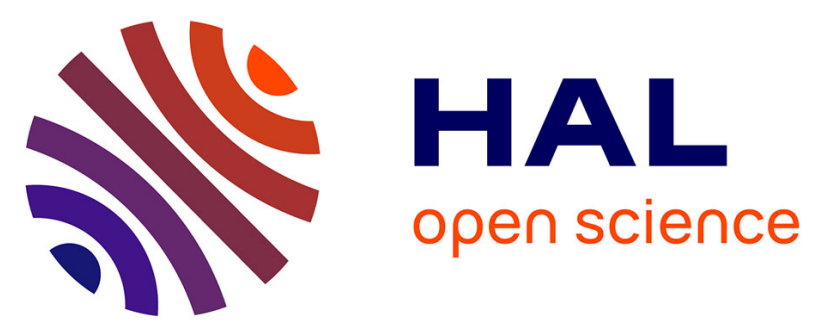

\title{
Rejection of sliding effects in car like robot control: application to farm vehicle guidance using a single RTK GPS sensor
}

\author{
R. Lenain, B. Thuilot, C. Cariou, P. Martinet
}

\section{- To cite this version:}

R. Lenain, B. Thuilot, C. Cariou, P. Martinet. Rejection of sliding effects in car like robot control: application to farm vehicle guidance using a single RTK GPS sensor. 2003 IEEE/RSJ International Conference on Intelligent Robots and Systems, Oct 2003, Las Vegas, Ethiopia. pp.3811-3816, 10.1109/IROS.2003.1249748 . hal-02466492

\section{HAL Id: hal-02466492 \\ https://hal.inria.fr/hal-02466492}

Submitted on 4 Feb 2020

HAL is a multi-disciplinary open access archive for the deposit and dissemination of scientific research documents, whether they are published or not. The documents may come from teaching and research institutions in France or abroad, or from public or private research centers.
L'archive ouverte pluridisciplinaire HAL, est destinée au dépôt et à la diffusion de documents scientifiques de niveau recherche, publiés ou non, émanant des établissements d'enseignement et de recherche français ou étrangers, des laboratoires publics ou privés. 


\title{
Rejection of sliding effects in car like robot control : application to farm vehicle guidance using a single RTK GPS sensor
}

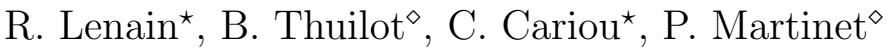 \\ * Cemagref \\ $\diamond$ LASMEA \\ BP 50085 - 24, av. des Landais \\ 24, av. des Landais \\ 63172 Aubière Cedex France \\ 63177 Aubière Cedex France \\ roland.lenain@cemagref.fr \\ Benoit.Thuilot@lasmea.univ-bpclermont.fr
}

\begin{abstract}
A very accurate vehicle guidance is required in numerous agricultural applications, as seeding, spraying, row cropping, ... Accuracy in vehicle localization can be obtained in realtime from a RTK GPS sensor. Several control laws, relying on this sensor, have been previously designed and provide satisfactory results as long as vehicles do not slide. However, sliding has to occur in agricultural tasks (sloping fields, curves on a wet land, ...). The challenge addressed in this paper is to preserve vehicle guidance accuracy in such situations. A nonlinear adaptive control law is here designed. Simulation results and field experiments, demonstrating the capabilities of that control scheme, are reported and discussed.
\end{abstract}

Keywords: mobile robots, nonlinear control systems, adaptive control laws, kinematic GPS, agriculture.

\section{INTRODUCTION}

RTK GPS (also named kinematic GPS) provides realtime localization with a centimeter accuracy. Since these sensors are available, many works on vehicle guidance using this technology have been designed. Numerous applications deal with agricultural tasks. The advantages of automatic guidance in this area are obvious: it reduces the tiring driving task and increases both precision and productivity in the agronomic work carried out. Moreover, the sensor appears here very suitable: since agricultural vehicles move far from buildings or trees, interruptions in GPS signal reception (which is the major concern with this sensor) do not occur. Several guidance devices have already been marketed by specialized companies (AgSystems, Integrinautics, ...), GPS systems suppliers (Trimble, ...) or agricultural manufacturers (John Deere, ...) However, the above mentioned devices, as well as works reported in the literature, make use of several sensors: RTK GPS and inertial sensors in [7], multiple RTK GPS antennas in [8], RTK GPS, vision and NIR in [9], .. Moreover, they are mainly concerned with straight lines following on even ground. In previous works, we have addressed the twofold challenge: relying on a unique RTK GPS sensor and providing vehicles with curved path fol- lowing capabilities. Our solution, reported in [11], consists in a nonlinear control law, taking into account for the reference path curvature, and a Kalman state reconstructor, providing the whole vehicle state vector from the information delivered from the unique RTK GPS sensor. Satisfactory experiments have been carried out on dry and even grounds. We have however noticed that as soon as vehicles move in a slippery environment (sharp curves on a wet ground, sloping fields, ...), guidance accuracy is degraded: a lateral deviation appears, and could be important, depending on the sliding conditions.

In this paper, control algorithms presented in [11] are modified, relying on adaptive control techniques, in order to reject sliding effects on the vehicle behavior. Preliminary results presented in [6] are refined, and extensive simulations and experimentations are discussed.

\section{STEERING LAW IN ABSENCE OF SLIDING}

\section{A Notations}

The vehicle, see Figure 1, is described according to Ackermann's model, also named bicycle model: front and rear axles are simplified to two virtual wheels (one front and one rear). This model is quite common in mobile robot control theory, when non sliding is assumed, see for instance [12]. Our notations are now detailed (see also Figure 1)

- $\mathcal{C}$ is the path to be followed,

- $O$ is the center of vehicle virtual rear wheel,

- $M$ is the point on $\mathcal{C}$ which is the closest to $O$.

$M$ is assumed to be unique, which is realistic when the vehicle remains quite close from $\mathcal{C}$.

- $s$ is the curvilinear coordinate of point $M$ along $\mathcal{C}$, and $c(s)$ denotes the curvature of $\mathcal{C}$ at that point.

- $y$ and $\tilde{\theta}$ are respectively lateral and angular deviation of the vehicle with respect to reference path $\mathcal{C}$ (see Figure 1).

- $\delta$ is the virtual front wheel steering angle.

- $v$ is the vehicle linear velocity, considered here as a parameter, whose value may be time-varying during the vehicle evolution.

- $L$ is the vehicle wheelbase. 


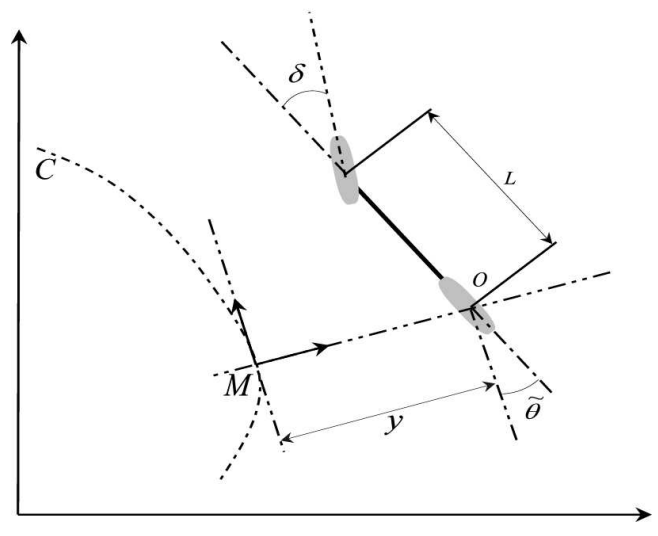

Figure 1: Vehicle modeling parameters

\section{$B$ Vehicle kinematic model}

Vehicle configuration is completely specified when $s, y$ and $\tilde{\theta}$ are given. These 3 variables can then be seen as a vehicle state vector. Under non sliding assumption, their evolution can be shown to obey the following kinematic equations (see e.g., [12], [11], ...):

$$
\left\{\begin{aligned}
\dot{s} & =\frac{v \cos \tilde{\theta}}{1-c(s) y} \\
\dot{y} & =v \sin \tilde{\theta} \\
\dot{\tilde{\theta}} & =v\left(\frac{\tan \delta}{L}-\frac{c(s) \cos \tilde{\theta}}{1-c(s) y}\right)
\end{aligned}\right.
$$

Velocity $v$ is here considered as a parameter: the only control variable is $\delta$. It is assumed hereafter that $c(s) y \neq 1$ (i.e lateral deviation does not bring vehicle point $O$ on path $\mathcal{C}$ curvature center): it is always satisfied during actual path following, since the vehicle remains close to $\mathcal{C}$.

\section{Steering law design}

It has been established that most of mobile robots kinematic models can be converted, without any approximation, into almost linear models named chained forms, see for instance [10]. Such an approach is attractive, since control laws can then be designed according to Linear Systems Theory, while still relying upon the actual nonlinear mobile robots kinematic models.

More precisely, nonlinear kinematic model is first converted into chained form via invertible state and control nonlinear transformations. A linear control law is then designed relying on the chained form. Finally, the actual control law is computed via state and control nonlinear transformations. In [11], this technique is applied to kinematic model (1). It is shown that curved path following (i.e. maintaining $y$ and $\tilde{\theta}$ equal to 0 ) can be achieved according to nonlinear control law:

$$
\begin{aligned}
\delta(y, \tilde{\theta})= & \arctan \left(L \left[\frac { \operatorname { c o s } ^ { 3 } \tilde { \theta } } { ( 1 - c ( s ) y ) ^ { 2 } } \left(\frac{d c(s)}{d s} y \tan \tilde{\theta}\right.\right.\right. \\
& -K_{d}(1-c(s) y) \tan \tilde{\theta}-K_{p} y \\
& \left.\left.\left.+c(s)(1-c(s) y) \tan ^{2} \tilde{\theta}\right)+\frac{c(s) \cos \tilde{\theta}}{1-c(s) y}\right]\right)
\end{aligned}
$$

where $K_{p}$ and $K_{d}$ can be interpreted as parameters of a $\mathrm{PD}$ controller.

\section{$D$ Experimental equipment}

Experiments have been carried out with an ARES 640 RENAULT-Agriculture tractor, shown on Figure 2. The RTK GPS receiver is a Thales Navigation dual frequency "Aquarius 5002" unit, which supplies an absolute position with a $\pm 2 \mathrm{~cm}$ accuracy, at a $10 \mathrm{~Hz}$ sampling frequency.

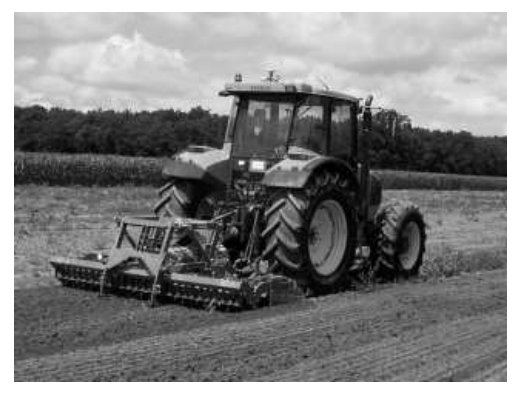

Figure 2: Experimental vehicle

The mobile antenna has been located on the top of the cabin, straight up the point $O$. Therefore, current value of state variable $y$ is inferred from direct position measurements. Current value of $\tilde{\theta}$ can be inferred, under non sliding assumption, from point $O$ velocity measurements, also provided by the RTK GPS sensor. However, experiments have shown that values of $\tilde{\theta}$ thus obtained are indeed very noisy. Therefore, raw velocity measurements are here proceeded through a Kalman state reconstructor, see [11]. Obviously, this approach could provide data which are not always relevant for sliding detection, as it will be seen hereafter.

\section{E Vehicle behavior in presence of sliding}

Steering law (2) provides satisfactory results, as long as no sliding occurs during experiments, see [11]. However, farm vehicles move on fields which do not always propose good adherence properties: sliding can indeed be noticed when vehicles enter into curves, or on sloping fields. Steering law (2) is then strongly disturbed and lateral deviation with respect to the reference path can be observed.

As an example, Figure 3 presents lateral deviation recorded during straight line following on a sloping field. Since slope, and therefore sliding conditions, are constant, lateral deviation converges (on an average) to a constant non null value. It can also be shown that heading deviation and steering angle also converge to non null values.

\section{MODEL INCORPORATING SLIDING EFFECTS}

Sliding effects can be taken into account, either by designing a vehicle dynamic model, or by introducing perturbations inside the kinematic model previously designed under non sliding assumption. In this paper, the second 


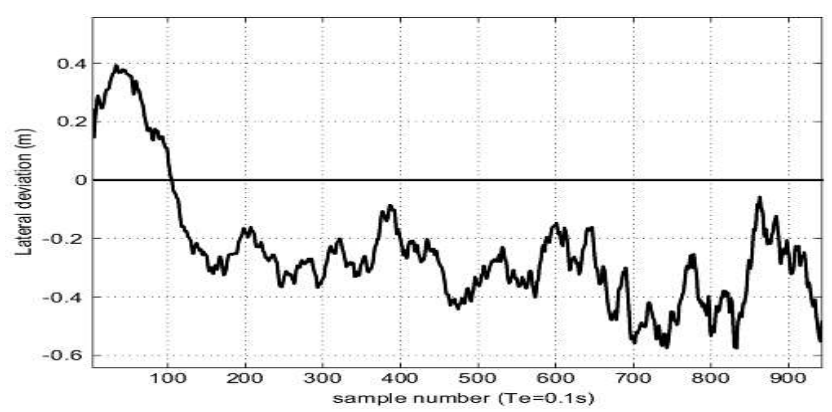

Figure 3: Vehicle behavior in presence of sliding

alternative has been considered since, on one hand it allows to still rely, for a large part, on previously designed steering law (2), and on the other hand dynamic models of agricultural vehicles do not appear very tractable from the control point of view: adherence conditions are varying, and their online identification is difficult. Dynamic models are also dependent on the vehicle configuration, and especially on the implement being used. Such difficulties are related in [4], where dynamic control is investigated. Refining kinematic models in order to account for sliding effects has also been proposed, with an approach different from ours, in [1]. It has also been used in nautical applications, in order to account for current effects, see [5].

\section{A Sliding description}

Vehicle dynamics analysis, see [3], identifies sliding effects as the superposition of two motions:

- Translation: when the four tires slide in the same direction (e.g. when the vehicle moves along a slope), a lateral motion occurs.

- Rotation: in Ackermann's model (see Figure 1), the vehicle is described by a front and a rear wheel. When sliding occurs, the two virtual wheels do not slide exactly in the same way (due to mass repartition, tire structure, ...). This difference generates a rotation motion.

It can be accounted for these two motions by introducing two sliding variables, namely $\dot{Y}_{p}$ and $\dot{\Theta}_{p}$, in model (1) previously designed under non sliding assumption:

$$
\left\{\begin{aligned}
\dot{s} & =\frac{v \cos \tilde{\theta}}{1-c(s) y} \\
\dot{y} & =v \sin \tilde{\theta}+\dot{Y}_{p} \\
\dot{\tilde{\theta}} & =v\left(\frac{\tan \delta}{L}-\frac{c(s) \cos \tilde{\theta}}{1-c(s) y}\right)+\dot{\Theta}_{p}
\end{aligned}\right.
$$

Such a model is representative exclusively of lateral motion due to sliding effect. In our application, longitudinal sliding has not been integrated since it has no influence on control accuracy: on one hand, it acts only on vehicle velocity which is considered as a parameter of model (3), and on the other hand steering law performances (2) have been shown to be independent from $v$ (see [11]).
$B$ Estimation of sliding variables

Sliding variables $\dot{Y}_{p}$ and $\dot{\Theta}_{p}$ can be online estimated by comparing the actual evolution of the vehicle, assumed to obey equations (3), and its theoretical evolution in absence of sliding, which can be simulated from model (1). This estimation approach, depicted on Figure 4, leads then to the following relations:

$$
\left\{\begin{array}{c}
\dot{Y}_{P}=\frac{y^{k}-y^{k-1}}{T_{e}}-v \sin \tilde{\theta}^{k-1} \\
\dot{\Theta}_{P}=\frac{\theta^{k}-\theta^{k-1}}{T_{e}}-v \frac{\tan \delta^{k-1}}{L}
\end{array}\right.
$$

where $T_{e}$ denotes the sampling period, and $y^{k}, \theta^{k}$ and $\delta^{k}$ stand for the values of the lateral deviation $y$, the absolute vehicle heading $\theta$ and the steering angle $\delta$ at sample time $k T_{e}$. Relations (4) are very convenient, since sliding variables are not computed from absolute lateral and heading deviations, but from relative deviations measured for a sampling period. Sliding estimation is therefore independent from the vehicle absolute deviation.

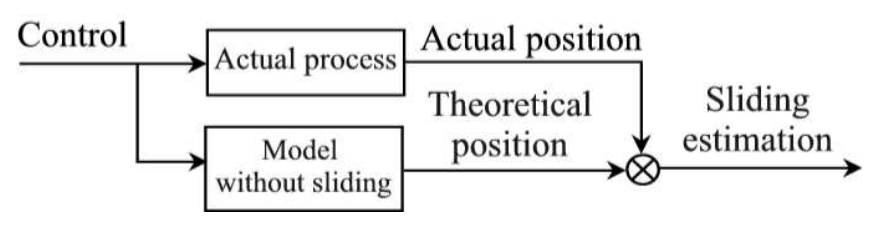

Figure 4: Sliding estimation

\section{Experimental validation}

In order to evaluate both sliding modeling and estimation, the following experiments have been carried out:

- Several path following have been performed on slippery grounds with steering law (2). The experimental vehicle is the farm tractor depicted on Figure 2.

- The evolutions of sliding variables $\dot{Y}_{p}$ and $\dot{\Theta}_{p}$ during these experiments, have then been computed according to relations (4).

- Next, these actual evolutions of $\dot{Y}_{p}$ and $\dot{\Theta}_{p}$ have been reported in vehicle model (3) in order to simulate tractor trajectories.

- Relevance of model (3) is finally investigated by comparing the actual and simulated tractor trajectories.

Figure 5 shows the 3 paths that have been followed:
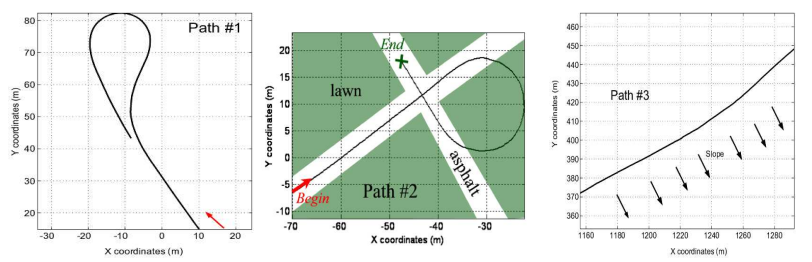

Figure 5: Paths to be followed

- Path \#1 : the vehicle moves on a graveled ground and performs a half turn. Sliding occurs during the curve. 
- Path \#2 : this path is built on different kinds of ground: straight line parts take place on asphalt whereas a part of the curve is drawn on lawn.

- Path \#3 : the trajectory consists mainly in a straight line on a sloping wet field (slope is indicated via arrows on Figure 5). No implement is towed.

Figures 6, 7 and 8 display that simulated lateral deviation in presence of sliding is consistent with actual measurements. Nevertheless, several points can be pointed out:

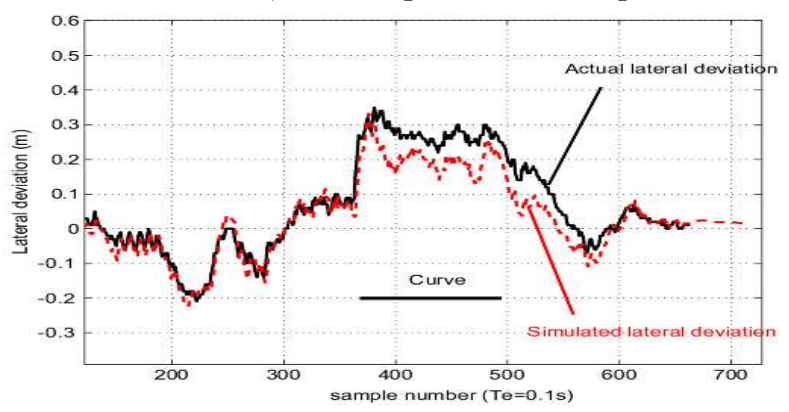

Figure 6: Path\#1 following with steering law (2)

First, we notice that lateral deviation is slightly underestimated during a curve following (see for instance Figure 6 during steady curvature - from iterations 375 up to 500). These misestimations are mainly due to the Kalman filter used to evaluate angular deviation $(\tilde{\theta})$ as it was discussed in "Experimental equipment". Since the presence of a filter introduces some delay, angular sliding variable $\left(\dot{\Theta}_{P}\right)$ is inevitably lowered during the curve. A posteriori computations corroborate this fact : by using a shifted angular deviation information to compute sliding variables, simulation results on lateral deviation fit more precisely to actual deviations observed $( \pm 5 \%)$. Differences between simulated and actual evolution are less important on path\#2 and path\#3 following (see Figures 7 and 8), because ground properties (lawn for path\#2 and path\#3) or path to be followed (straight line in path\#3) make influence of $\tilde{\theta}$ in sliding evaluation less important than on graveled ground and/or during a curved path following.

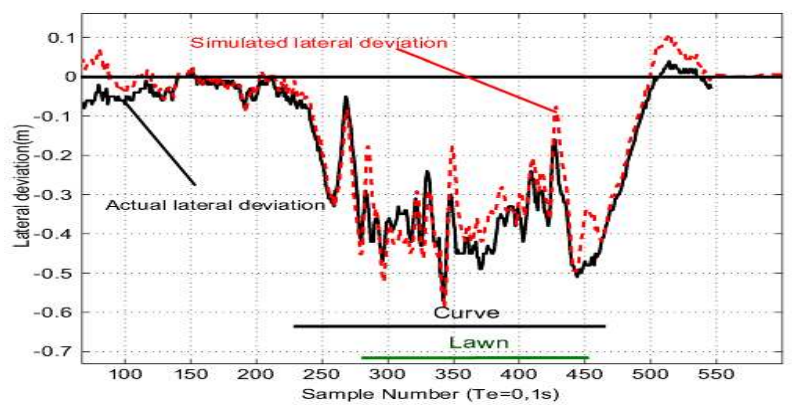

Figure 7: Path\#2 following with steering law (2)

Figures 7 and 8 display quite large oscillations in simu- lated lateral deviation. They can be related to the pendular motion undergone by the mobile GPS antenna: since it is located on the top of the tractor cabin, ground irregularities generate, via the shock absorber linking axles to fairing, a lateral motion of the antenna, which is wrongly interpreted as a sliding motion. Simulation of model (3) therefore generates lateral deviations which do not exist in the actual experiment.

Perturbations due to such pendular movements are amplified when the path to be followed takes place on a non planar ground. Figure 7 displays clearly the difference when the vehicle evolutes on asphalt and on lawn. On iterations up to 270 and after 450, lateral deviation is quite smooth whereas between iterations from 270 to 450 , lateral deviation is strongly noisy. This points out that sliding variables will have to be filtered out prior to their use in control law (described hereafter), even if some delay is then introduced. Nevertheless, Figures 6 to 8 show that sliding effects are satisfactorily described by model (3), which can be used to design an adaptive control law.

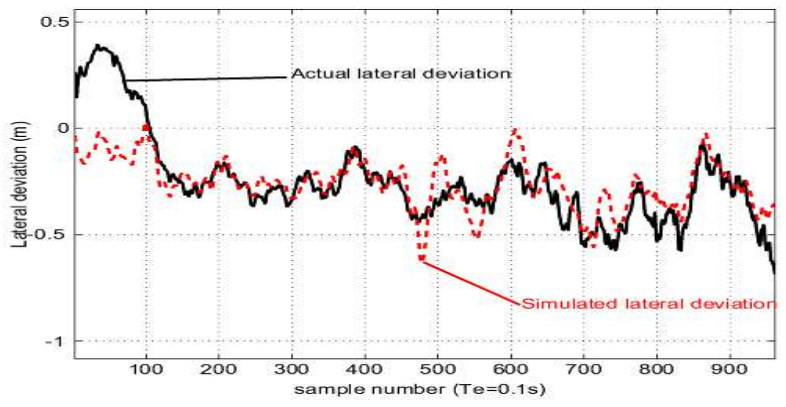

Figure 8: Path\#3 following with steering law (2)

\section{ADAPTIVE CORRECTION TECHNIQUES}

\section{A Adaptive control law design}

In order to introduce the principle of the adaptive control law, let us first consider a theoretical situation, where sliding variables $\dot{Y}_{p}$ and $\dot{\Theta}_{p}$ are constant. It can then be shown that steering law (2) (designed under non sliding assumption) leads to constant guidance errors. More precisely, reporting (2) in model (1), and neglecting second order terms in $y$ prove that (see also [6]):

$$
y \stackrel{t \rightarrow \infty}{\longrightarrow}-\frac{\beta+\frac{\dot{\Theta}_{p}}{v \cos ^{3} \tilde{\theta}}}{\alpha-\frac{2 c(s) \dot{\Theta}_{p}}{v \cos ^{3} \tilde{\theta}}} \triangleq y_{c}
$$

with :

$$
\left\{\begin{array}{rll}
\tilde{\theta} & \stackrel{t \rightarrow \infty}{\longrightarrow}-\arcsin \left(\frac{\dot{Y}_{p}}{v}\right) \\
\delta & \stackrel{t \rightarrow \infty}{\longrightarrow} \arctan L\left(-\frac{\dot{\Theta}_{p}}{v}+\frac{c(s) \cos \tilde{\theta}}{1-c(s) y}\right) \\
\alpha & =\frac{d c(s)}{d s} \tan \tilde{\theta}+c(s) \tan \tilde{\theta}\left(K_{d}-c(s) \tan \tilde{\theta}\right)-K_{p} \\
\beta & =\tan \tilde{\theta}\left(c(s) \tan \tilde{\theta}-K_{d}\right)
\end{array}\right.
$$


These results corroborate that $\tilde{\theta}$ and $\delta$ converge also to non null values, indicating that the vehicle moves crabways. Moreover, it can be observed that rotational sliding $\dot{\Theta}_{p}$ acts on steering angle $\delta$, when translational sliding $\dot{Y}_{p}$ acts on heading deviation.

If $c(s)$ was also constant, it is then immediate to prove that lateral deviation can be brought back to 0 by simply shifting the objective of steering law (2): if its objective is to bring $y$ to the constant value $-y_{c}$, then $y$ will converge to 0 in presence of constant sliding. Steering law (2) writes then:

$$
\begin{aligned}
\delta= & \arctan \left(L \left[\frac { \operatorname { c o s } ^ { 3 } \tilde { \theta } } { ( 1 - c ( s ) ( y + y _ { c } ) ^ { 2 } } \left(\frac{d c(s)}{d s}\left(y+y_{c}\right) \tan \tilde{\theta}\right.\right.\right. \\
& -K_{d}\left(1-c(s)\left(y+y_{c}\right)\right) \tan \tilde{\theta}-K_{p}\left(y+y_{c}\right) \\
& \left.\left.\left.+c(s)\left(1-c(s)\left(y+y_{c}\right)\right) \tan ^{2} \tilde{\theta}\right)+\frac{c(s) \cos \tilde{\theta}}{1-c(s) y}\right]\right)
\end{aligned}
$$

In practical situations, sliding variables are not constant. However, if the shift in the steering law objective (i.e. $y_{c}$ ) could track the current sliding conditions, then steering law (6) would still improve guidance accuracy. Two approaches are hereafter proposed to determine online the value of $y_{c}$ :

- online simulation: the vehicle model incorporating sliding effects (i.e. model (3)), fed by the current sliding variables $\dot{Y}_{p}$ and $\dot{\Theta}_{p}$ (computed from (4)), and controlled via steering law (2), is simulated online. The state variable $y$ of that simulator is then used as the corrective shift $y_{c}$ to be introduced in the actual control law (6) steering the vehicle.

When $\dot{Y}_{p}$ and $\dot{\Theta}_{p}$ are constant, the simulated state variable $y$ obviously converges to the expected shift value. When they are not constant, the simulated state variable $y$ always reflects the current sliding conditions.

- direct computation: the corrective shift $y_{c}$ to be introduced in the actual control law (6) is directly computed by reporting the current sliding variables $\dot{Y}_{p}$ and $\dot{\Theta}_{p}$ in relation (5).

When $\dot{Y}_{p}$ and $\dot{\Theta}_{p}$ are constant, the corrective shift is then immediatly the suitable one. This second approach may then appear more relevant. However, it is more sensitive to noise and perturbations than the first one, where the simulator acts as a natural lowpass filter. In practical situations, the corrective shift computed from relation (5) will have to be filtered out. Therefore, none of these approaches appears clearly superior to the other.

The overall adaptive control scheme is depicted on Figure 9 , where the adaptive module consists either in online simulation or direct computation. In the general adaptive control framework, it can be seen as a model-based adaptive control scheme.

\section{$B$ Simulation results}

Performances of adaptive steering law (6) have first been investigated through simulations. The kernel of the sim-

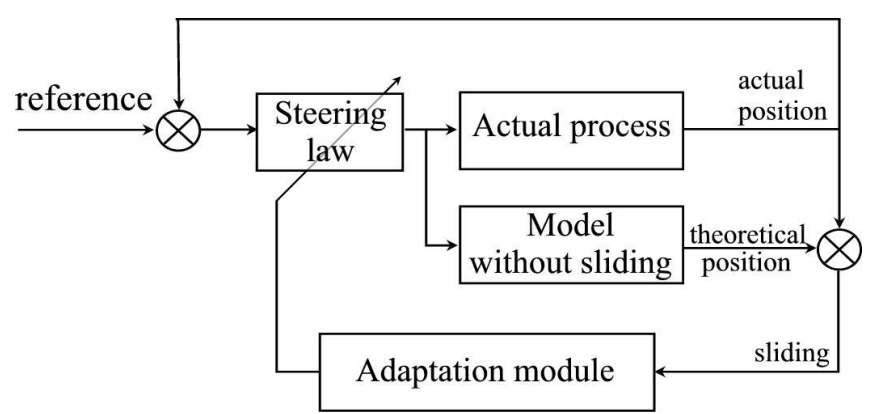

Figure 9: Overall adaptive control scheme

ulator (replacing the actual vehicle) accounts for sliding effects, just as model (3), but has been written with absolute variables, instead of lateral and heading deviations. The evolution of the sliding variables $\dot{Y}_{p}$ and $\dot{\Theta}_{p}$ is those recorded in the 3 experiments displayed on Figures 6 to 8 . The overall control scheme is depicted on Figure 9. Sliding estimation encloses a subsimulator, which does not account for sliding effects (sliding estimation module is also displayed separately on Figure 4). The two adaptation modules have been investigated. The first one, named online simulation, encloses a second subsimulator, which accounts for sliding effects, as above mentioned. The lateral deviation obtained with each adaptation module is compared on Figures 10 and 11 with those obtained without adaptive correction (i.e. with steering law (2)).

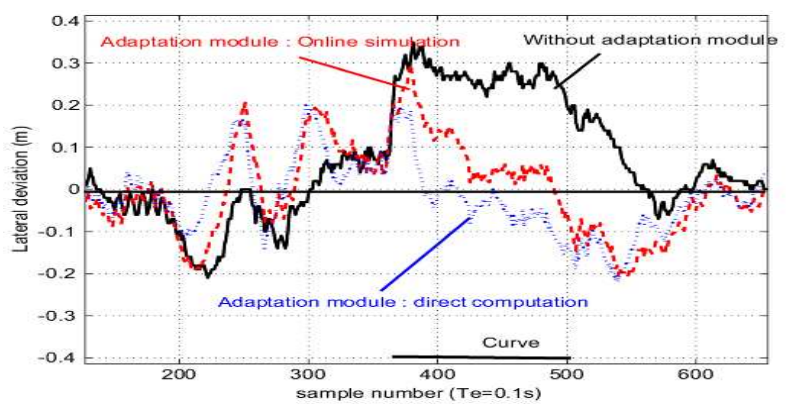

Figure 10: Simulation of path\#1 following with law (6)

Figure 10 shows path \# 1 following. Sliding occurs as soon as the vehicle enters into the curve: it is just as if a step was applied on sliding variables $\dot{Y}_{p}$ and $\dot{\Theta}_{p}$. It can be observed that adaptive control law relying on the direct computation adaptation module reacts faster than those relying on the online simulation adaptation module. This is quite natural since the cutoff frequency of the lowpass filter used in the former adaptation module is here higher than the natural cutoff frequency of the subsimulator used in the latter module. During the curve, since sliding variables are then almost constant, lateral deviation with both adaptive laws converges to 0 , as expected. Finally, at the end of the curve (as at beginning), lat- 
eral deviation presents some non null values, pointing out some delay in the settling of the appropriate corrective shift $y_{c}$.

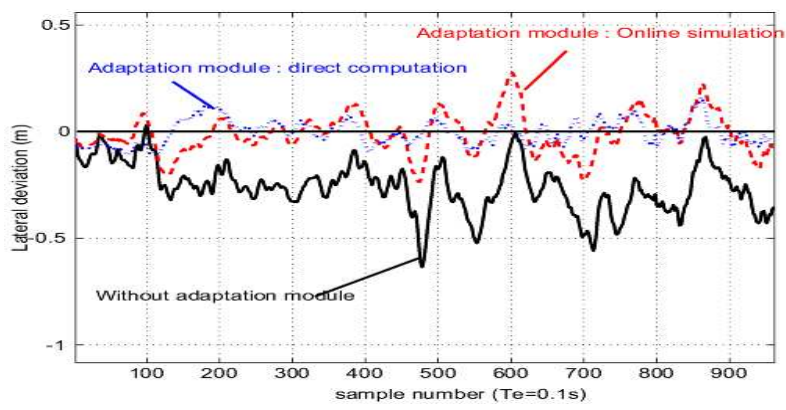

Figure 11: Simulation of path\#3 following with law (6)

Figure 11 shows path \# 3 following, i.e. straight line following on a sloping field. The slope of the actual field, and therefore the sliding variables $\dot{Y}_{p}$ and $\dot{\Theta}_{p}$ used in these simulations, are not perfectly constant. It can be observed that the corrective shift $y_{c}$ does not evolve fast enough to keep lateral deviation constantly equal to 0 . Nevertheless, the mean value of lateral deviation is close to zero, which is not the case when adaptive correction is not used.

\section{Experimental results}

These simulation results have been corroborated by experiments. Figure 12 compares the lateral deviations measured when the farm tractor achieves path \#1 following with or without adaptation module. Actual deviations

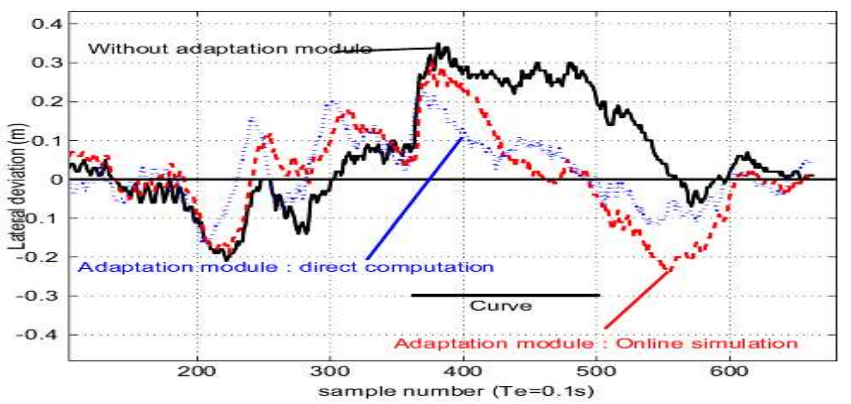

Figure 12: Experiments of path\#1 following with law (6)

appear quite similar to those obtained in simulation, see Figure 10. It can just be noticed that lateral deviation overshoots, at the beginning and at the end of the curve, are larger than in simulation. They proceed from delays pointed out when discussing Figures 6 to 8. Moreover, delays introduced by low level actuators amplify also these overshoots. Nevertheless, it must be pointed that during the curve, both adaptive control laws bring back the vehicle on the reference trajectory, when the non adaptive one leads to a constant lateral deviation.

\section{CONCLUSION AND PROSPECTS}

In this paper, an adaptive control scheme, relying on model-based techniques, is proposed to take into account for sliding effects when steering car like mobile robots. The adaptive control law nevertheless still relies on the nonlinear steering law previously designed, under non sliding assumption, using chained systems theory. All the advantages of this nonlinear control technique are then preserved. Capabilities of the proposed adaptive scheme have been validated, both in simulations and experiments. Remaining difficulties deal with delays in the sensing system and in the low level actuation. A heading GPS sensor is currently integrated, in order to reduce delays in vehicle heading measurements, and improve their robustness. Predictive control techniques are also being studied, using a priori models, to reduce overshoots observed during curved path following. Finally, another sliding model has been developed. It still relies on mobile robot chained forms designed under non sliding assumption. The capabilities of the adaptive laws designed with this second model are presently compared to those presented here.

\section{References}

[1] Ackermann J.. Robust Lateral and Yaw Control. In Proc. of Eur. summer school in automatic control, Grenoble (France), 2002.

[2] Borne P., Dauphin-Tanguy G., Rotella F. and Zambettakis I. Commande et optimisation des processus. Series : Méthodes et pratiques de l'ingénieur, TECHNIP Editions, Paris (France) 1990.

[3] Dormegnie E., Fandard G., Mahajoub G., Zarka F.. Dynamique du véhicule. Lectures at French Institute for Advanced Mechanics (IFMA), 2002.

[4] Ellouze M. and d'Andréa-Novel B. Control of unicycle-type robots in the presence of sliding effects with only absolute longitudinal and yaw velocities measurement. In European Journal of Control, 6:567-584, 2000.

[5] Holzhüter T. and Schultze R. Operating experience with a high-precision track controller for commercial ships. In Control Engineering Practice 4(3):343-350, 1996.

[6] Lenain R., Thuilot B., Cariou C. and Martinet P. Adaptive control for car like vehicles guidance relying on RTK GPS: rejection of sliding effects in agricultural applications. In Proc. of the Intern. Conf. on Robotics and Automation (ICRA), Taipei, Sept. 2003.

[7] Nagasaka Y., Otani R., Shigeta K. and Taniwaki K. Automated operation in paddy fields with a fiber optic gyro sensor and GPS. In Proc. of the Intern. Workshop on Robotics and Automated Machinery for Bio-Productions (Bio-Robotics), pp 21-26, Valencia (Spain) September 1997.

[8] O'Connor M., Elkaim G., Bell T. and Parkinson B. Automatic steering of a farm vehicle using GPS. In Proc. of the $3^{\text {rd }}$ Intern. Conf. on Precision Agriculture, Minneapolis (USA), pp 767777, June 1996.

[9] Ried J. and Niebuhr D. Driverless tractors. In Ressource 8(9):7-8, September 2001.

[10] Samson C. Control of chained systems. Application to path following and time-varying point-stabilization of mobile robots. In IEEE Trans. on Automatic Control 40(1):64-77, January 1995.

[11] Thuilot B., Cariou C., Martinet P. and Berducat M.. Automatic guidance of a farm tractor relying on a single CP-DGPS. In Autonomous robots 13(1):53-71, July 2002.

[12] The Zodiac. Theory of robot control. Canudas de Wit C., Siciliano B. and Bastin G. eds, Springer Verlag, Berlin (Germany) 1996. 